\title{
Perfil de seleção e consumo de medicamentos em uma instituição de ensino superior: diagnóstico situacional para promoção da saúde
}

\section{| ${ }^{1}$ Jaqueline Rocha Borges dos Santos, ${ }^{2}$ Patrícia Seda Gomes de Castro I}

Resumo: A seleção de medicamentos é um processo interdisciplinar baseado no perfil epidemiológico, econômico e técnico local. Os medicamentos selecionados devem apresentar eficácia e segurança, de acordo com a Política Nacional de Medicamentos. Este trabalho teve como objetivo identificar o perfil de seleção e consumo de medicamentos dispensados em uma instituição de ensino superior. A partir de pesquisa descritiva com característica quantitativa e qualitativa, foram utilizados os mapas de dispensação de medicamentos para a enfermagem da Divisão de Saúde de 2018 e 2019. Em 2018, 21\% dos medicamentos padronizados não foram consumidos. Por esta razão, em 2019 houve nova seleção e 17,2\% dos medicamentos foram excluídos. De acordo com o perfil de consumo de medicamentos, observou-se que o consumo de analgésicos foi superior ao consumo de fármacos com propósito anti-inflamatório, de acordo com a classificação Anatomical Therapeutic Chemical (ATC). Conclui-se que o perfil abordado neste estudo possibilita um diagnóstico situacional de seleção e consumo, tendo como necessidade a implantação do seguimento farmacoterapêutico com o intuito de minimizar as possíveis reações adversas produzidas por medicamentos, como estratégia de prevenção e promoção da saúde.

> Palavras-chave: seleção de medicamentos; medicamentos essenciais; uso de medicamentos; promoção da saúde.

\author{
' Departamento de Ciências \\ Farmacêuticas, Universidade \\ Federal Rural do Rio de Janeiro. \\ Seropédica-RJ, Brasil (jaqueroc \\ jr@gmail.com). ORCID: 0000- \\ 0002-2429-5689 \\ 2 Universidade Federal Rural do \\ Rio de Janeiro. Seropédica-RJ, \\ Brasil (pattyseda19@gmail.com). \\ ORCID: 0000-0001-9213-3713
}

Recebido em: 05/01/2020 Aprovado em: 18/09/2020 Revisado em: 06/10/2020 


\section{Introdução}

A seleção de medicamentos é um processo interdisciplinar que consiste na escolha do medicamento dentre todos os itens referendados pela Relação Nacional de Medicamentos Essenciais (RENAME), tendo como critério o perfil epidemiológico, econômico e técnico. Nos níveis de atenção à saúde, a Comissão de Farmácia e Terapêutica (CFT) exibe um papel estratégico na seleção de medicamentos, com o propósito de garantir segurança, eficácia e custo-efetividade no Sistema Único de Saúde (SUS), tendo como objetivo estabelecer as condutas terapêuticas e direcionar o processo de aquisição. A seleção de medicamentos é importante, pois a partir dela são estabelecidas as etapas da assistência farmacêutica (AF) (WEBER et al., 2010; SFORSIN et al., 2012).

O medicamento é considerado uma tecnologia em saúde utilizado, quando necessário, no processo de cuidado. Para tanto, está implicado em avaliaçóes quanto à eficácia, segurança e custo-efetividade em processos de tomada de decisão (SILVA; ELIAS, 2019). Soma-se a isto a necessidade de correlacionar com o perfil de usuários. Neste trabalho, destaca-se a comunidade acadêmica representada por estudantes que, majoritariamente, buscam no cotidiano os serviços de saúde de uma universidade pública brasileira.

Considerando o perfil socioeconômico dos estudantes de graduação da Universidade Federal Rural do Rio de Janeiro (UFRRJ), 78\% apresentam renda familiar per capita de até 1,5 salário mínimo, com 32\% participando de programas do Plano Nacional de Assistência Estudantil (Pnaes), divididos em: 16,2\% beneficiários do auxílio alimentação e 14,9\% beneficiários do auxílio-moradia (ANDIFES, 2019). Estes dados reforçam a necessidade de políticas institucionais em saúde para atender à população estudantil, articuladas com as políticas públicas em saúde, com destaque para a temática medicamentos. A garantia dos princípios do SUS, aliada às medidas de prevenção, promoçâo e recuperação da saúde devem ser os norteadores de açôes vinculadas à Divisão de Saúde da referida instituição federal de ensino superior (IFES).

\section{Política Nacional de Medicamentos}

A Política Nacional de Medicamentos (PNM), aprovada em outubro de 1988, tem como objetivo garantir a eficácia, segurança e qualidade dos medicamentos, promover o uso racional e garantir o acesso da população aos medicamentos essenciais. 
De acordo com a percepçáo de gestores da AF, o perfil de consumo medicamentoso para o planejamento em AF é relacionado com os indicadores demográficos que mostram o aumento da expectativa de vida (TORRES et al., 2014). No entanto, os motivos que levaram à criaçáo da PNM foram o uso irracional de medicamentos, a falta de acesso da população aos medicamentos e os problemas com a qualidade dos medicamentos (BRASIL, 2006).

A PNM possui algumas diretrizes que buscam ações de reorientação da assistência farmacêutica, regulaçáo sanitária, garantir qualidade de medicamentos, o uso racional, capacitação de recursos humanos, dentre outras (PONTES JUNIOR et al., 2008). A PNM estabeleceu que os medicamentos essenciais devem ser selecionados a partir da RENAME e definiu que os estados e municípios devem elaborar suas próprias listas de medicamentos essenciais tendo esta como referência, sendo necessário investigar se a necessidade da seleção de medicamentos envolve o trabalho da CFT (MARQUES; ZUCCHI, 2006). Estados e municípios, em seus serviços de saúde, determinam quais medicamentos serão selecionados para compor a padronização, com base no perfil epidemiológico, nas prioridades escolhidas pela gestão e nos recursos financeiros disponibilizados para esta finalidade (FARIAS et al., 2016).

\section{Seleção e Relação Nacional de Medicamentos Essenciais (RENAME)}

A seleção de medicamentos se destaca nas etapas da assistência farmacêutica, pois permite elaborar uma lista de medicamentos para promover o uso racional de medicamentos (FARIAS et al., 2016). A elaboração de uma lista de medicamentos em nível nacional é realizada pela Comissão Nacional de Incorporação de Tecnologias no SUS (Conitec), que tem como finalidade assessorar o Ministério da Saúde (MS) na seleção de tecnologias, incorporação, exclusão ou inclusão de novos medicamentos pelo SUS (BRASIL, 2016).

Os medicamentos essenciais são indispensáveis para atender à necessidade da população. Esses medicamentos devem estar disponíveis nos segmentos da sociedade que deles necessitam (BRASIL, 2001). São selecionados de acordo com os medicamentos registrados no país. Durante a escolha dos medicamentos essenciais, estabelece-se um processo comparativo com os representantes da mesma classe terapêutica (BRASIL, 2012).Os fármacos que representam a mesma classe terapêutica são submetidos a 
análise, tendo como base os elementos comparativos, para que o escolhido determine o real e mais relevante benefício clínico ao paciente. Cada grupo farmacológico conta com vários representantes que possuem aspectos farmacocinéticos diferentes que repercutem favoravelmente na terapêutica. Esses representantes devem possuir eficácia e segurança, que são comprovadas por ensaios clínicos randomizados. $\mathrm{Na}$ maioria das vezes, os representantes são escolhidos por serem utilizados em mais de uma doença. Os medicamentos de alto custo não são excluídos da lista, desde que representem a melhor escolha para uma condição específica. Os medicamentos que possuem eficácia e segurança similar, introduzidos recentemente no mercado e com insuficiência de uso, eficácia e segurança não comprovada, devem ser excluídos da lista de medicamentos (WANNMACHER, 2006).

A seleção de medicamentos depende de alguns fatores, considerados determinantes sociais de saúde, como: características das doenças, fatores demográficos, fatores socioeconômicos, fatores biopsicossociais, condições de saneamento e moradia. Para tanto, considera-se em primeiro lugar a relação entre perfil epidemiológico e necessidade de medicamentos para o perfil determinado. Simultaneamente, utilizase como referência a RENAME, sendo que cada estado pode elaborar a Relação Estadual de Medicamentos Estaduais (RESME), que segue para o município, que define a Relação Municipal de Medicamentos Essenciais (REMUME).

Todos os medicamentos constantes na RENAME estão elencados no formulário terapêutico nacional e os medicamentos do componente especializado da AF estão definidos no contexto de Protocolos Clínicos e Diretrizes Terapêuticas (PCDT) elaborados pelo MS (BRASIL, 2020). Somado a isto, são consideradas características inerentes à composição e à formação da equipe de saúde, como: competência para trabalho interprofissional em saúde e tomada de decisão baseada em evidências científicas.

O comitê de especialidades da OMS estabeleceu alguns critérios para a seleção e uso de medicamentos essenciais, como a relação custo-eficácia, consideraçôes farmacocinéticas, locais de disponibilidade de instalações, fabricação e armazenamento. Além disso, os medicamentos devem estar dispostos em formas farmacêuticas que garantam qualidade e biodisponibilidade esperada, aliado ao fato de que a maioria dos medicamentos essenciais devem conter apenas um princípio ativo (WHO, 2002a). 
Em 1997, a Organização Mundial da Saúde (OMS) criou o modelo de listas de medicamentos essenciais para que os países possam criar suas próprias listas (WHO, 2002b). A RENAME visa satisfazer os cuidados com a saúde da população e tem como vantagem reduzir os custos devido a procura limitada de medicamentos, estimulando a eficácia deste sistema e melhorando a disponibilidade do medicamento a nível nacional; e ainda, melhora a utilização do medicamento pelo paciente, já que o número de medicamentos disponíveis e prescritos são reduzidos (BRASIL, 2017).

A Comissão de Revisão da RENAME investiga, analisa e seleciona os medicamentos que devem estar presentes, correlacionando com as necessidades da população a partir do perfil epidemiológico. Esta comissão é composta por diversos profissionais: médicos, farmacêuticos, enfermeiros, além de organizaçôes não governamentais, dentre outros. Esses profissionais são responsáveis pela seleção de medicamentos essenciais para o país. Dentre as atribuições desta comissão está a avaliação para inclusão de novos medicamentos substituindo os medicamentos já existentes, a partir de evidências comprovadas de benefício clínico.

A RENAME é dividida por grupos terapêuticos. Estes são divididos por subgrupos, que por sua vez estáo divididos em subclasses que estáo dispostas em ordem alfabética, de acordo com a Denominação Comum Brasileira (DCB) ou a Denominação Comum Internacional (DCI); contendo a forma farmacêutica, a dose e a indicação terapêutica (BRASIL, 2017).

\section{O uso da classificação Anatomical Therapeutic Chemical (ATC)}

A ATC (Anatomical Therapeutic Chemical), sigla que significa "Anatômica Terapêutica Química”, forma o sistema ATC/DDD, em conjunto com a Dose Diária Definida (Defined Daily Dose). Esse sistema passou a ser reconhecido pela OMS como um padrão internacional de utilização de fármacos no ano de 1996. "A" é uma unidade de medida de consumo de medicamentos, sendo definida para cada fármaco e refletindo na dose diária média de cada fármaco, de acordo com sua principal indicação (TODESCATO; BERTOLOTO; MELLO, 2017; VIDOTTI, 1993).

A classificação ATC tem como objetivo proporcionar dados estatísticos da utilização de medicamentos e comparação internacional, para garantir a qualidade com o uso de medicamentos, tendo como componente a comparação estatística do consumo de fármacos. O princípio da classificação ATC é monitorar o uso racional e irracional de medicamentos (WHO, 2019). 
O primeiro passo da metodologia ATC é a introdução dos códigos ATC em todos os fármacos. O uso do sistema ATC permite a padronização de grupos de medicamentos e a comparação do uso de fármacos entre países, regióes e locais que proporcionam assistência à saúde (WHO, 2019). Os medicamentos podem possuir um ou mais códigos ATC, dependendo de sua dose e formulação com uso terapêutico diferente. As estatísticas de consumo funcionam como base para realizar avaliaçôes econômicas para a saúde, sendo importante monitorar o consumo de medicamentos, publicar mais informaçôes sobre os novos fármacos introduzidos no mercado e seus efeitos, além de planejar estudos considerados hábitos de prescriçôes, a fim de melhorar as decisôes políticas de saúde no que se refere ao uso de medicamentos (VIDOTTI, 1993).

O uso da classificação ATC tem como vantagens a atualização anual, a identificação do principal uso terapêutico, a existência de um único código para determinado medicamento; no entanto, podem ser utilizados diferentes códigos ATC para uma substância presente em duas ou mais concentraçôes e formulaçóes com utilização terapêutica diferente (TODESCATO; BERTOLOTO; MELLO, 2017).

No sistema de classificação ATC, as substâncias ativas são divididas em cinco níveis diferentes. No primeiro, encontram-se os grupos anatômicos/farmacológicos. Cada grupo principal tem o segundo nível, dividido em farmacológicos ou terapêuticos; já o terceiro e quarto níveis são divididos em subgrupos químicos, farmacológicos ou terapêuticos. Por fim, o quinto nível é representado pela substância química (WHO, 2019).

\section{A seleção de medicamentos na Divisão de Saúde da UFRRJ}

Segundo a Deliberação n ${ }^{\circ}$ 51, de 22 de abril de 1981, da Universidade Federal Rural do Rio de Janeiro, a Divisão de Saúde tem como objetivo proporcionar assistência médica de emergência aos estudantes e definindo a terapêutica clínica adequada; bem como encaminhar o paciente para outra instituição de saúde ou para outro nível de atenção à saúde, caso a necessidade de saúde não esteja inserida dentre suas atividades. Localizada no município de Seropédica, a Divisão de Saúde conta com uma farmacêutica responsável pela compra de medicamentos, controle de estoque, controle de saída e entrada de medicamentos, dispensação, seleção, além de orientação aos pacientes quanto ao uso racional de medicamentos, articulada com outros profissionais da saúde (UFFRJ, 1981). 
A seleção de medicamentos na Divisão de Saúde da UFRRJ é realizada pela farmacêutica responsável, que analisa o perfil de consumo de medicamentos, estabelecendo uma lista de medicamentos essenciais. Essa lista contém os medicamentos selecionados de acordo com o perfil epidemiológico relacionado com a demanda de procura do serviço de saúde, tendo como princípio padronizar os medicamentos existentes, proporcionando o uso racional de medicamentos e garantindo a farmacoterapia adequada para reduzir os possíveis problemas relacionados com medicamentos.

A seleção de medicamento é importante para o seguimento farmacoterapêutico, tendo como objetivo escolher os medicamentos essenciais e com efetividade terapêutica para incluir na lista de medicamentos da Divisão de Saúde. Além de os medicamentos apresentarem uma efetividade terapêutica, devem exibir baixa toxicidade para garantir uma boa qualidade de vida para o paciente.

Deste modo, o objetivo do presente trabalho foi identificar o perfil de dispensação de medicamentos na Divisão de Saúde da Universidade Federal Rural do Rio de Janeiro (UFRRJ), aos pacientes atendidos no serviço de saúde, para a realização do seguimento farmacoterapêutico como estratégia de prevenção e promoção da saúde. Nesse contexto, destacam-se: (1) identificação dos medicamentos constantes na Divisão de Saúde da UFRRJ; (2) análise do consumo mensal de medicamentos na Divisão de Saúde da UFRRJ; (3) relação do consumo de medicamentos com a sazonalidade; (4) relação do perfil dos medicamentos existentes na Divisão de Saúde da UFRRJ com os grupos sistêmicos da classificação ATC; (5) identificação dos medicamentos de maior consumo na Divisão de Saúde da UFRRJ; e (6) relação das reaçôes adversas ocasionadas pelos fármacos de maior consumo com a necessidade do seguimento farmacoterapêutico.

\section{Materiais e Métodos}

Realizou-se pesquisa descritiva com característica quantitativa e qualitativa, utilizando como técnica para coleta de dados os mapas de saída de medicamentos para a enfermagem que apresentam os nomes de todos os medicamentos usados na Divisão de Saúde da UFRRJ, de $1^{\circ}$ de março de 2018 a 31 de maio de 2019.

O estudo foi realizado por meio de uma coleta de dados com a ajuda da farmacêutica que disponibilizou os mapas que são utilizados como controle para 
saída de medicamentos. Esses mapas contêm a lista de todos os medicamentos padronizados, número de medicamentos presentes no estoque atual, reposição e o total de saída semanal.

Na primeira etapa do estudo, realizou-se levantamento do consumo mensal dos medicamentos mais utilizados na Divisão de Saúde da UFRRJ, de $1^{\circ}$ de março de 2018 a 31 de maio de 2019, tendo como base os mapas de controle, nos quais são anotados todos os medicamentos dispensados para a enfermagem durante a semana ou diariamente. Os mapas foram separados por mês e realizou-se a contagem de saída total de cada medicamento. A partir disso, foi elaborada tabela dos medicamentos mais utilizados, dividida por grupos de medicamentos disponíveis na Divisão de Saúde da UFRRJ. Os medicamentos foram assim divididos: 1) medicamentos injetáveis; 2) medicamentos Orais; e 3) medicamentos inalatórios.

$\mathrm{Na}$ segunda etapa, foi estabelecida uma correlação entre a sazonalidade de consumo. Na terceira, os medicamentos foram divididos conforme os grupos sistêmicos e grupos terapêuticos da classificação ATC. A classificação ATC é dividida por grupos que são representados por letras, cada letra indica um grupo anatômico (WHO, 2019). Após, foram calculados o consumo médio e a frequência (\%) dos medicamentos mais utilizados no período de estudo. Na quarta etapa, foram analisadas as reaçóes adversas que os medicamentos de maior consumo podem ocasionar, correlacionando essas informaçóes com a necessidade de propor um seguimento farmacoterapêutico.

\section{Resultados e Discussão}

Na Divisão de Saúde da UFRRJ, foram analisados 162 mapas de dispensação de medicamento para a enfermagem, de $1^{\circ}$ de março de 2018 a 31 de maio de 2019.

\section{Caracterização dos mapas de dispensação de medicamentos para a enfermagem}

Os mapas de dispensação de medicamentos eram gerados mensalmente em 2018, e diariamente em 2019. Durante a coleta de dados, foram obtidos 11 mapas de saída de medicamento para a enfermagem no ano de 2018. Estes apresentavam 79\% de medicamentos injetáveis, $17,7 \%$ de medicamentos orais e 3,4\% de medicamentos inalatórios. Também foram analisados 151 mapas de saída de medicamento para 
a enfermagem no ano de 2019, que apresentavam a quantidade de saída diária, sendo $86 \%$ de medicamentos injetáveis, 9,6\% medicamentos orais e 3,8\% de medicamentos inalatórios.

Os mapas apresentam todos os medicamentos selecionados pela farmacêutica na Divisão de Saúde da UFRRJ, sendo que no ano de 2018 havia 62 medicamentos e em 2019 o total de medicamentos selecionados foi de 53. No ano de 2018, foram selecionados 49 medicamentos injetáveis, 11 orais e dois inalatórios. No ano de 2019, houve decréscimo no número de medicamentos injetáveis e orais, passando a conter então 45 medicamentos injetáveis e seis orais (tabela 1).

Tabela 1. Quantidade de formas farmacêuticas dos medicamentos selecionados nos mapas de dispensação para a enfermagem na Divisão de Saúde da UFRRJ nos anos de 2018 e 2019. Seropédica-RJ, 2019

\begin{tabular}{c|c|c}
\hline Medicamentos & Total (2018) & Total (2019) \\
\hline Injetáveis & 49 & 45 \\
\hline Orais & 11 & 2 \\
\hline Inalatórios & 2 & 53 \\
\hline TOTAL & 62 & 6 \\
\hline
\end{tabular}

Fonte: elaboração própria.

No ano de 2019, a Divisão de Saúde da UFRRJ diminuiu o quadro de medicamentos orais selecionados e padronizados, considerando que a administração deveria ser avaliada como, de fato, necessária. Em comparação com os medicamentos selecionados do ano de 2018 com 2019, permaneceram 47 (73,4\%), foram excluídos $11(17,2 \%)$ e incluídos seis $(9,4 \%)$ medicamentos nos mapas de saída para a enfermagem (tabela 2). A farmacêutica selecionou quais medicamentos de administração oral iriam permanecer e os que deveriam ser excluídos na lista de medicamentos essenciais da Divisão de Saúde. A seleção de medicamentos orais ocorreu devido à falta de uma farmácia ambulatorial para fazer a dispensação de medicamentos orais - por exemplo, comprimidos e cápsulas. Por esse motivo, o quadro de medicamentos orais presentes é menor se comparado aos injetáveis. 
Tabela 2. Levantamento dos medicamentos, quanto à forma farmacêutica, selecionados que permaneceram, foram incluídos e excluídos nos mapas de 2019. Seropédica-RJ, 2019

\begin{tabular}{c|c|c|c}
\hline Medicamentos & Permanentes & Incluídos & Excluídos \\
\hline Injetáveis & 39 & 6 & 6 \\
\hline Orais & 6 & 0 & 5 \\
\hline Inalatórios & 2 & 0 & 0 \\
\hline TOTAL & 47 & 6 & 11 \\
\hline
\end{tabular}

Fonte: elaboração própria.

Observou-se que na Divisão de Saúde da UFRRJ, em 2019, 17,2\% dos medicamentos foram excluídos e 9,4\% incluídos (tabela 2) na lista de medicamentos, comparando com a lista do ano anterior. Os medicamentos são selecionados de acordo com o perfil epidemiológico, que pode ser analisado pelo consumo médio anual de cada medicamento. Os medicamentos que apresentaram menor consumo médio ou baixa dispensação foram provavelmente excluídos ou substituídos por outros medicamentos da mesma classe terapêutica com a garantia de eficácia, qualidade e menor custo.

Para realizar a inclusão de medicamentos, deve ser evitada a multiplicidade dos princípios ativos que possuem a mesma finalidade, assim como as associaçóes medicamentosas. O propósito é selecionar e padronizar medicamentos com um único princípio ativo, para que os médicos possam associar os medicamentos necessários nas doses definidas para cada caso. Além disso, devem ser consideradas a eficácia, a segurança e a baixa toxicidade.

Na Divisão de Saúde da UFRRJ, todos os medicamentos selecionados estão presentes na lista de medicamentos essenciais da RENAME, que é atualizada periodicamente. Porém, 21\% dos medicamentos padronizados no ano de 2018 não foram consumidos. Por esta razão, realizou-se seleção de medicamentos no ano de 2019, tendo como base o consumo de medicamentos do ano anterior. A farmacêtica responsável retirou todos os medicamentos que não exibiam demanda para utilização e estabeleceu um estoque mínimo para os medicamentos que tinham baixo consumo.

\section{Consumo de medicamentos do mês de março a dezembro do ano de 2018}

A partir da avaliação do consumo médio de medicamentos na Divisão de Saúde, verificou-se que $20,7 \%$ correspondiam ao mês de março e abril, $25,9 \%$ ao mês 
de maio e junho, $12,6 \%$ julho e agosto, $13,7 \%$ setembro e outubro; e no mês de novembro e dezembro, obteve-se o maior percentual de consumo, 27,2\% (figura 1).

De acordo com a sazonalidade, notou-se que o consumo nos meses de julho e agosto reduziu $47 \%$ comparado aos outros meses do ano (figura 1). Nesse período, os estudantes da UFRRJ encontram-se de férias e aproximadamente $12 \mathrm{mil}$ estudantes universitários que "não residem" retornam para suas cidades de origem, permanecendo por um tempo prolongado no município de Seropédica apenas no período acadêmico letivo (PREFEITURA DE SEROPÉDICA, 2014).

Observou-se que nos meses de maio e junho o consumo de diclofenaco sódico, dipirona, succinato sódico de hidrocortisona e fosfato dissódico de dexametasona são maiores do que nos meses de novembro e dezembro (figura 2). Nos meses de maio e junho é outono, que tem como características as quedas bruscas na temperatura e a diminuição do período de chuva, denotando um clima seco, com a possibilidade da manifestação de doenças respiratórias (inflamatórias ou alérgicas) como asma e rinite (VIEIRA, 2017; NUNES et al., 2016). Assim, o consumo de dipirona, diclofenaco sódico, succinato sódico de hidrocortisona e fosfato dissódico de dexametasona aumentou nesse período, possibilitando o tratamento adequado para as doenças sazonais predominantes da estação. A dipirona pode ser utilizada como antitérmico e analgésico, aliviando os sintomas de dor e febre.

Figura 1. Consumo bimestral de medicamentos na Divisão de Saúde da UFRRJ de março a dezembro de 2018. NV/DZ - novembro/dezembro; ST/OU - setembro/outubro; JL/AG - julho/agosto; MR/AB - março/abril; MA/JN - maio/junho.

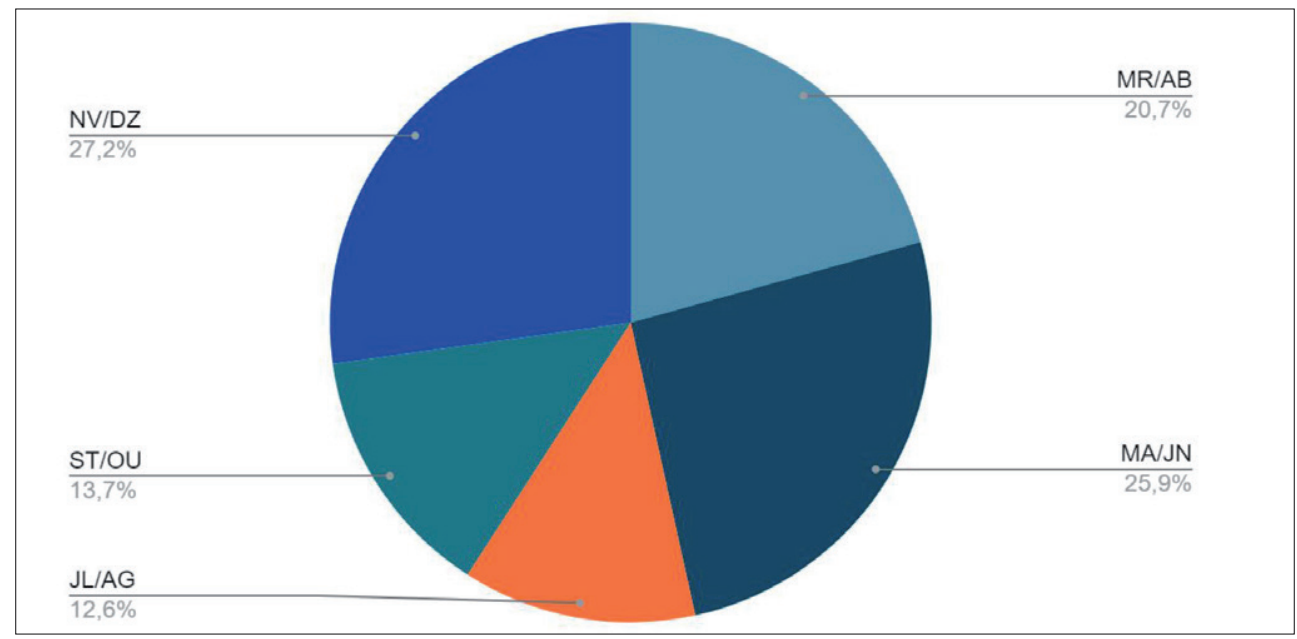


Figura 2. Consumo de medicamentos nos meses de maio/junho e novembro/ dezembro de 2018. NV/DZ - novembro/dezembro; MA/JN - maio/junho.

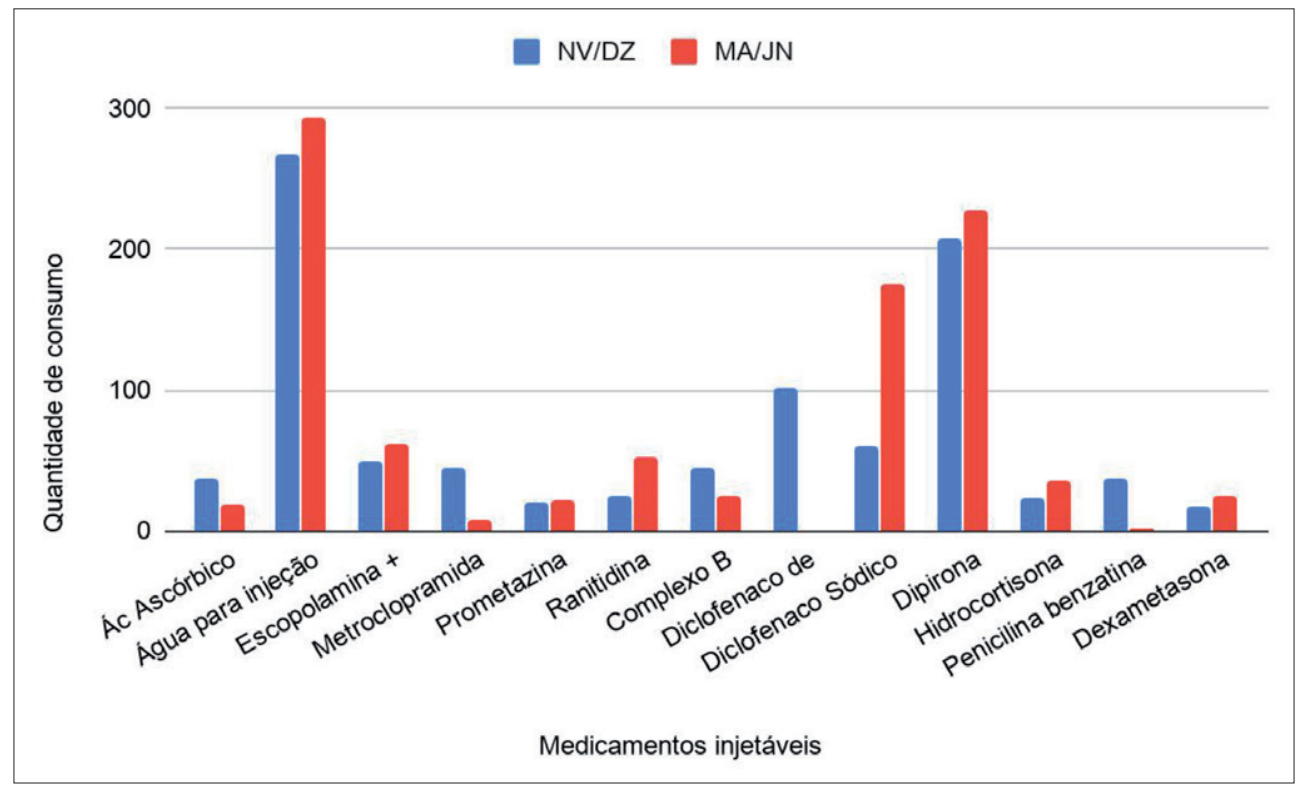

\section{Medicamentos mais utilizados na Divisão de Saúde da UFRRJ, correlacionando com a classificação ATC}

A tabela 3 apresenta os medicamentos mais utilizados de acordo com o consumo médio e a frequência. Com isso, avalia-se que a água para injeção tem o maior consumo médio comparado ao consumo dos 62 medicamentos selecionados, apresentando frequência de 25,3\%. Em seguida aparecem dipirona $1 \mathrm{mg} / 2 \mathrm{~mL}$ $(15,9 \%)$, diclofenaco sódico (9,7\%) e cloreto de sódio $9 \%$ frasco com $500 \mathrm{~mL}(9,1 \%)$.

$\mathrm{Na}$ Divisão de Saúde da UFRRJ, a maioria dos medicamentos que compóem a lista de medicamentos é injetável. Este fato justifica o grande consumo de água para injeção. 
Tabela 3. Consumo médio e porcentagem de consumo dos 22 fármacos e solução hipotônica mais prescritos e consumidos em 2018 na Divisão de Saúde da UFRRJ. CM - consumo médio. CM - consumo médio. Porc - porcentagem. Seropédica-RJ, 2019

\begin{tabular}{|c|c|c|c|c|}
\hline Medicamentos injetáveis & Código ATC & Total & $\mathrm{CM}$ & Porc $(\%)$ \\
\hline Ácido Ascórbico $100 \mathrm{mg} / \mathrm{mL}$ & A11GA01 & 87 & 17,4 & 1,9 \\
\hline Água para injeção $10 \mathrm{~mL}$ & V07AB00 & 1.148 & 229,6 & 25,3 \\
\hline Bromoprida $10 \mathrm{mg}$ & A03FA04 & 93 & 18,6 & 2,1 \\
\hline $\begin{array}{l}\text { Butilbrometo de Escopolamina + } \\
\text { Dipirona }\end{array}$ & $\begin{array}{c}\text { A04AD01, } \\
\text { N05CM05, S01FA02 }\end{array}$ & 236 & 47,2 & 5,2 \\
\hline Cloreto de Potássio 10\% & A12BA01 & 64 & 12,8 & 1,4 \\
\hline Cloreto de sódio $0,9 \%$ Frasco $500 \mathrm{~mL}$ & B05XA02 & 413 & 82,6 & 9,1 \\
\hline Cloreto de sódio 0,9\% Frasco $10 \mathrm{~mL}$ & B05XA03 / R01AX10 & 69 & 13,8 & 1,5 \\
\hline Cloridrato de Metoclopramida & A03FA01 & 135 & 27 & 3 \\
\hline Cloridrato de Prometazina 50mg/ $2 \mathrm{ml}$ & R06AD02 & 87 & 17,4 & 1,9 \\
\hline Cloridrato de Ranitidina $25 \mathrm{mg} / \mathrm{mL}$ & A02BA02 & 157 & 31,4 & 3,5 \\
\hline Complexo B & A11EA00 & 103 & 20,6 & 2,3 \\
\hline Diclofenaco de Potássio & M01A B05 & 117 & 23,4 & 2,6 \\
\hline Diclofenaco Sódico & M01AB05 & 439 & 87,8 & 9,7 \\
\hline Dipirona $1 \mathrm{mg} / 2 \mathrm{~mL}$ & N02BB02 & 722 & 144,4 & 15,9 \\
\hline $\begin{array}{l}\text { Fosfato Dissódico de Dexametasona } \\
4 \mathrm{mg} / \mathrm{mL}\end{array}$ & D07AB19 & 62 & 12,4 & 1,4 \\
\hline Furosemida $10 \mathrm{mg} / \mathrm{mL}$ & C03CA01 & 63 & 12,6 & 1,4 \\
\hline Glicose de 5\% & B05BA03 & 117 & 23,4 & 2,6 \\
\hline Glicose de $25 \%$ & B05BA04 & 101 & 20,2 & 2,2 \\
\hline Hidrocortisona $500 \mathrm{mg}$ & H02АВ09 & 106 & 21,2 & 2,3 \\
\hline Penicilina benzatina 1.200 .000 UI & J01CE08 & 83 & 16,6 & 1,8 \\
\hline Ácido acetilsalicílico 100 mg & B01AC06 & 64 & 12,8 & 1,4 \\
\hline Captopril $25 \mathrm{mg}$ & C09AA01 & 67 & 13,4 & 1,5 \\
\hline TOTAL & & 4.533 & 906,6 & 100 \\
\hline
\end{tabular}

Fonte: elaboração própria. 
Dos 62 medicamentos mais solicitados através dos maiores grupos sistêmicos da classificação ATC, os mais utilizados na Divisão de Saúde no período de estudo pertencem ao Grupo V (Vários), com frequência de 23,8\%, B (Sangue e Sistema Hematológico) 18,1\% e o Grupo A (Trato Alimentar e Metabolismo) 17,4\% (tabela 4). Vale completar que o grupo $V$ é representado por alérgenos, restante de produtos terapêuticos, agentes de diagnóstico, nutrientes gerais, restante de produtos não terapêuticos, meios de contraste, radiofármacos de diagnóstico, radiofármacos terapêuticos e pensos cirúrgicos (WHO, 2019).

Tabela 4. Quantidade e porcentagem de consumo dos 62 medicamentos mais solicitados segundo os grandes grupos sistêmicos da classificação ATC. Seropédica-RJ, 2019

\begin{tabular}{l|c|c}
\hline \multicolumn{1}{c|}{ Classificação ATC $1^{\circ}$ nível } & Quantidade & Porcentagem de Consumo (\%) \\
\hline A (Trato Alimentar e Metabolismo) & 838 & 17,4 \\
\hline B (Sangue e Sistema Hematológico) & 875 & 18,1 \\
\hline C (Sistema cardiovascular) & 185 & 3,8 \\
\hline H (Preparações Hormonais) & 110 & 2,3 \\
\hline J (Anti-infecciosos de Uso Sistêmico) & 97 & 11,9 \\
\hline M (Sistema Músculo- Esquelético) & 573 & 17,1 \\
\hline N (Sistema Nervoso) & 823 & 3,7 \\
\hline R (Sistema Respiratório) & 177 & 23,8 \\
\hline V (Vários) & 1.148 & 100 \\
\hline TOTAL & 4.826 & \\
\hline
\end{tabular}

Fonte: elaboração própria.

A tabela 5 apresenta o segundo nível de classificação ATC dos medicamentos utilizados na Divisão de Saúde, correlacionando os principais grupos terapêuticos existentes com a quantidade de medicamentos consumidos e a frequência (\%). Nota-se que o nível V07 (restante de produtos não terapêuticos), representando 23,8\%, foi o mais consumido, seguido pelos níveis B05 (Substitutos do sangue e soluções de perfusão), com 16,8\%; N02 (Analgésicos), com 16,3\% e M01 (Produtos antirreumáticos e anti-inflamatórios), com 11,9\%. 
Tabela 5. Consumo e porcentagem de consumo dos 62 medicamentos mais solicitados segundo os grandes grupos terapêuticos da classificação ATC. Seropédica-RJ, 2018

\begin{tabular}{|c|c|c|}
\hline Classificação ATC $2^{\circ}$ nível & Quantidade & $\begin{array}{c}\text { Porcentagem de } \\
\text { Consumo (\%) }\end{array}$ \\
\hline A02 - (Antiácidos) & 160 & 3,3 \\
\hline A03 - (Agentes antiespasmódicos, anticolinérgicos) & 230 & 4,8 \\
\hline A04 - (Antieméticos e antinauseantes) & 258 & 5,3 \\
\hline A11 - (Vitaminas) & 190 & 3,9 \\
\hline B01 - (Agentes antitrombóticos) & 64 & 1,3 \\
\hline B02 - (Anti-hemorrágicos) & 2 & 0,1 \\
\hline B05 - (Substitutos do sangue e soluçôes de perfusão) & 809 & 16,8 \\
\hline C01 - (Terapia cardíaca) & 55 & 1,1 \\
\hline C03 - (Diuréticos) & 63 & 1,3 \\
\hline C09 - (Agentes que agem no sistema renina-angiotensina) & 67 & 1,4 \\
\hline H02 - (Corticoides de uso sistêmico) & 110 & 2,3 \\
\hline J01 - (Antimicrobianos de uso sistêmico) & 97 & 2 \\
\hline M01 - (Produtos antirreumáticos e anti-inflamatórios) & 573 & 11,9 \\
\hline N02 - (Analgésicos) & 789 & 16,4 \\
\hline N03 - (Antiepiléticos) & 5 & 0,1 \\
\hline N05 - (Psicolépticos) & 29 & 0,6 \\
\hline R01 - (Preparações nasais) & 69 & 1,4 \\
\hline $\begin{array}{l}\text { R03 - (Antiasmáticos ou p/ tratar doenças obstrutivas das } \\
\text { vias aéreas) }\end{array}$ & 21 & 0,4 \\
\hline R06 - (Anti-histamínicos de uso sistêmico) & 87 & 1,8 \\
\hline V07 - (Restante de produtos não terapêuticos) & 1.148 & 23,8 \\
\hline TOTAL & 4.826 & 100 \\
\hline
\end{tabular}

Fonte: elaboração própria.

Dentre os medicamentos mais consumidos, conforme o segundo nível da classificação ATC, há os denominados "restante de produtos não terapêuticos, analgésicos e anti-inflamatórios”. No segundo nível da classificação ATC, os grupos terapêuticos de maior consumo foram V07 e o B05. O grupo V07 representa o 
denominado "restante de produtos não terapêuticos", como é o caso da água para injeção, que possui alto consumo, por ser considerado um diluente utilizado para o preparo das soluçôes injetáveis. Já no grupo B05 está presente o cloreto de sódio 0,9\%, utilizado para o restabelecimento de fluidos e eletrólitos; conhecido como soro fisiológico, é uma solução isotônica, cuja concentração de água é igual a das células (RATTI et al., 2011).

O analgésico mais utilizado foi a dipirona, que pertence ao grupo N (Sistema Nervoso) e o anti-inflamatório mais consumido foi o diclofenaco sódico, classificado no primeiro nível da ATC como grupo M (Sistema Músculo-Esquelético). Esses medicamentos estão presentes na lista de medicamentos do ano de 2019, tendo um estoque maior, devido à alta demanda de saída, assim como a água para injeção que pertence ao grupo V.

Segundo os resultados de Monje et al. (2019), com estudos realizados em um hospital, os anti-inflamatórios não esteroides (AINE) corresponderam a 37,8\%, e os analgésicos e antipiréticos a 46,2\% do consumo total no ano de 2015. A análise do consumo de analgésicos é essencial, pois permite monitorar o uso na prática clínica. A implantação de um comitê de controle da dor deve ser considerada, com o objetivo de desenvolver protocolos para uso racional de analgésicos, bem como sessóes de educaçáo sobre a dor e auditorias para verificar a qualidade do tratamento para a dor (MONJE et al., 2019). Os analgésicos constituem um dos grupos de amplo consumo, por serem usados para tratar o alívio da dor; são de fácil aquisição e parte deles são de venda livre (TIERLING et al., 2004).

De acordo com os dados apresentados neste estudo, os analgésicos e antipiréticos representam 16,3\% do consumo, sendo maior se comparado aos anti-inflamatórios que representam 11,9\%. Cabrita et al. (2001) também apontaram os analgésicos e antipiréticos com 16,7\% e os anti-inflamatórios com 12,2\% como os mais consumidos dentre 649 estudantes de oito cursos de graduação da Universidade de Lisboa. Estes dados são semelhantes aos identificados em nosso estudo. De modo complementar, os dados de Vosgerau et al. (2011) sinalizam que dipirona é o analgésico mais consumido em uma Unidade de Saúde da Família, considerado para alguns estudos o principal medicamento da terapêutica brasileira por corresponder a $31,8 \%$ do mercado (VALE, 2006). Outro estudo realizado no município de Itaocara, estado do Rio de Janeiro, analisando dados sobre a dispensação de 12 princípios ativos, demonstrou que dipirona obteve a maior dispensação, correspondendo a 45,9\%; já 
a dispensação de diclofenaco sódico foi de 8,99\% (NUNES et al., 2016). Estudos adicionais colocam o alto consumo per capita de analgésicos em vários países e a tendência de consumo dos analgésicos (DIENER; SCHNEIDER; AICHER, 2008; MLYNARZ-HIDER; CAVALIÉ; MAISON, 2018).

Ao considerar correlação com estudos semelhantes em contexto universitário, Lee et al. (2016) colocam a necessidade de garantia da prestação de cuidados preventivos adequados para jovens adultos e ao enfrentamento de disparidades em grupos desfavorecidos, por meio da ampliação da cobertura para os cuidados preventivos de saúde. Ainda de acordo com os autores, é fundamental estabelecer uma fonte habitual de cuidado, com foco em grupos vulneráveis e o desenvolvimento de diretrizes padronizadas de rastreamento de saúde para adultos jovens. Esta colocação reforça os achados de nosso estudo, sinalizando consumo de medicamentos para dor e inflamação que poderiam ser atenuados com ações de prevenção em saúde, aliado ao fato de que uma porcentagem elevada de estudantes da UFRRJ provém de famílias com baixa renda per capita (ANDIFES, 2019), corroborando a utilização dos serviços de saúde referenciados pelo SUS.

\section{Reaçóes adversas dos cinco medicamentos mais consumidos na Divisáo de Saúde da UFRRJ no período de estudo}

De acordo com o consumo de medicamentos na Divisão de Saúde da UFRRJ, o perfil de reaçôes adversas e as interaçôes medicamentosas foram analisados considerando os cinco medicamentos mais utilizados (tabela 6). O butilbrometo de escopolamina associado a dipirona, o cloridrato de metoclopramida e a dipirona em monoterapia podem causar hipotensão durante ou após a utilização, gerando queda da pressão arterial. Já o cloridrato de ranitidina pode ocasionar náusea, vômito, diarreia, cefaleia e sonolência, assim como o diclofenaco sódico (tabela 6) (LIMA et al., 2016; MANZI et al., 2011).

Os cinco medicamentos apresentados na tabela 6 não podem ser utilizados concomitantemente com alguns fármacos, podendo intensificar a ação desses ou diminuir a ação de ambos. Esses medicamentos não podem ser administrados com a ciclosporina, que é um imunossupressor, pois pode reduzir os níveis da ciclosporina. Esta não faz parte da lista de medicamentos da Divisão de Saúde, mas existem outros medicamentos que constam na lista e que náo podem ser associados a um desses cinco fármacos, como é o caso do ácido acetilsalicílico, que não pode ser utilizado juntamente 
com a dipirona, pois a esta pode reduzir o efeito do ácido acetilsalicílico na agregação plaquetária (PINHEIRO; WANNMACHER, 2010; JACOMINI; SILVA, 2011).

Observa-se que mesmo esses medicamentos apresentando alto consumo, as reaçôes adversas estão presentes, podendo causar risco à saúde do paciente. Por isso é de responsabilidade da equipe interprofissional de saúde orientar quanto ao uso adequado dos medicamentos, colaborando para a redução das reaçôes adversas e de automedicação. O acompanhamento ou seguimento farmacoterapêutico tem como objetivo detectar os resultados negativos associados à utilização de medicamentos (RNM), a partir de identificação dos problemas relacionados com medicamentos (PRM) (CAPUCHO, 2016).

Tabela 6. Algumas reaçóes adversas causadas pelos cinco medicamentos mais consumidos na Divisão de Saúde da UFRRJ. Seropédica-RJ, 2019

\begin{tabular}{l|l}
\hline \multicolumn{1}{c|}{ Medicamentos } & \multicolumn{1}{c}{ Reaçóes Adversas } \\
\hline $\begin{array}{l}\text { Butilbrometo de } \\
\text { escopolamina + dipirona }\end{array}$ & $\begin{array}{l}\text { Hipotensão, tontura, sonolência, pupilas dilatadas, pele seca, boca e } \\
\text { passagens respiratórias (SPINKS et al., 2007) }\end{array}$ \\
\hline $\begin{array}{l}\text { Cloridrato de } \\
\text { Metoclopramida }\end{array}$ & $\begin{array}{l}\text { Sonolência, sintomas extrapiramidais, diarreia, hipotensão } \\
\text { (VASCONCELLOS et al., 2014; OLSON et al., 2012) }\end{array}$ \\
\hline Cloridrato de Ranitidina & $\begin{array}{l}\text { Bradicardia, bloqueio atrioventricular, assistolia, reaçóes de } \\
\text { hipersensibilidade, pancreatite aguda, diarreia, náusea, vômitos, } \\
\text { constipaçáo, cefaleia, sonolência, insônia, vertigem e movimentos } \\
\text { involuntários reversíveis (MANZI et al., 2011; OLSON et al., 2012) }\end{array}$ \\
\hline Diclofenaco sódico & $\begin{array}{l}\text { Cefaleia, tontura, sonolência, vertigem, náusea, vômito, diarreia, } \\
\text { cólicas abdominais (RIBOLDI et al., 2012) }\end{array}$ \\
\hline Dipirona & $\begin{array}{l}\text { Reações hipotensivas transitórias isoladas, anemia aplástica, reaçóes } \\
\text { anafiláticas/anafilactoides (VALE, 2006; MANZI et al., 2011) }\end{array}$ \\
\hline
\end{tabular}

Fonte: elaboração própria.

No Brasil, os medicamentos são os principais causadores de intoxicaçôes, com 34,04\% dos casos registrados (SINITOX, 2016). Há também uma ausência de dados sobre o uso inadequado de medicamentos, como a ocorrência de reaçóes adversas e efetividade. Este fato ocorre devido à falta de conhecimento do paciente sobre o tratamento. Considerando o uso frequente de determinados medicamentos, grande parte da população não conhece as características essenciais para utilização dos mesmos com o mínimo de segurança (TIERLING et al., 2004). Com isso, é 
necessário que os profissionais da saúde informem os pacientes sobre os medicamentos de venda livre e os prescritos pelos médicos para colaborar com o uso econômico e racional dos mesmos (TIERLING et al., 2004).

Os medicamentos que não exercem o efeito terapêutico esperado se enquadram em inefetividade terapêutica. Esta pode ser considerada por alguns médicos como reaçôes adversas, mas pode se tratar de ausência ou diminuição do efeito associado à não adesão ao tratamento. Com isto, o seguimento farmacoterapêutico objetiva informar o paciente sobre o uso racional e seguro do medicamento, a fim de obter dados concretos que melhorem a qualidade de vida do paciente (CAPUCHO, 2016).

O diclofenaco sódico é utilizado para tratar dor e inflamação; pertence ao grupo dos AINE, que bloqueiam a síntese de prostaglandinas responsáveis pela de dor, inflamação e febre. Deve-se ter cautela para utilização de AINE em pacientes hipertensos, que utilizam anti-hipertensivos, devido à interação medicamentosa, em que o AINE diminui o efeito anti-hipertensivo (SOUZA et al., 2009). Somado a isto, estão as consequências gastrintestinais, assumindo reaçóes adversas como dor epigástrica, gastrite, náusea e refluxo (MANZI et al., 2011).

O cloridrato de ranitidina tem por objetivo reduzir a quantidade de ácido clorídrico produzido no estômago, devido à atuação antagonista nos receptores $\mathrm{H} 2$, favorecendo a melhora de quadros de gastrite, assim como de úlceras pépticas do estômago e do duodeno. Todavia, deve-se ter cautela em cardiopatas, devido à possibilidade de arritmia (CAVALCANTI et al., 2010). Somado a isto, vale o alerta para cenários de automedicação; uma vez que o uso contínuo pode mascarar problemas gastrintestinais preexistentes, além de colaborar para a redução da absorção de alguns nutrientes (LOPES; CARVALHO; FREITAS, 2010).

O cloridrato de metoclopramida é um fármaco antiemético, sendo utilizado no alívio dos sintomas de náuseas e vômito. Possui ação antidopaminérgica, melhorando o esvaziamento gástrico em curtos períodos de utilização. Seu uso deve ser realizado com cautela, pois pode causar irritabilidade, sonolência, manifestaçôes de efeitos extrapiramidais e eventos cardiovasculares (VASCONCELLOS; DUARTE; MACHADO, 2014).

O monitoramento contínuo do uso de medicamentos é fundamental para detectar os efeitos inesperados ou não conhecidos sejam identificados, avaliados, tratados e divulgados, sem causar danos à saúde da população. $\mathrm{O}$ monitoramento e o acompanhamento farmacoterapêutico são utilizados como estratégias, com o 
intuito de promover o uso racional de medicamentos, sendo mais efetivos quando há uma equipe interprofissional em saúde, trazendo benefícios clínicos e econômicos para o indivíduo e a sociedade (CAPUCHO, 2016).

\section{Conclusões}

A seleção de medicamentos na Divisão de Saúde da UFRRJ é uma prática que consiste na escolha dos medicamentos, tendo como referência o consumo, o perfil epidemiológico local e a eficácia dos medicamentos para garantir que os pacientes tenham o tratamento adequado. De acordo com os resultados obtidos, nota-se que os medicamentos com baixa ou nula dispensação foram excluídos ou substituídos por outros com baixo custo e a mesma propriedade terapêutica. Esta modificação aconteceu devido à nova seleçâo de medicamentos e à criação de uma nova lista de medicamentos.

Em consonância com a classificação ATC, de março a dezembro de 2018 houve maior consumo de analgésicos e anti-inflamatórios. A elevada dispensação dos medicamentos provenientes desses grupos terapêuticos se deve ao fato de a Divisão de Saúde ser classificada como um posto de saúde destinado à prevenção e à promoção da saúde. Logo, os casos mais graves são encaminhados à atenção secundária ou terciária à saúde.

A seleção e a padronização de medicamentos na Divisão de Saúde da UFRRJ devem atender às necessidades da populaçáo assistida. No que tange à garantia de segurança e eficácia, vale destacar que as reaçôes adversas devem ser monitoradas, assim como o conhecimento para orientação sobre interações medicamentosas. Estes aspectos sugerem a necessidade de implantação do seguimento farmacoterapêutico, articulado entre os outros profissionais da saúde, como estratégia de reduzir os problemas de saúde, bem como suas consequências secundárias, contribuindo para melhorar a qualidade de vida dos pacientes. ${ }^{1}$

\section{Referências}

ASSOCIAÇÃO NACIONAL DOS DIRIGENTES DAS INSTITUIÇÓES FEDERAIS DE ENSINO SUPERIOR. V Pesquisa nacional de perfil socioeconômico e cultural dos(as) graduandos(as) das IFES - 2018. Brasília: Fórum Nacional de Pró-reitores de Assuntos Comunitários e Estudantis (FONAPRACE), 2019. 318 p. Disponível em: <http://www.andifes. org.br/wp-content/uploads/2019/05/V-Pesquisa-Nacional-de-Perfil-Socioecon\%C3\%B4micoe-Cultural-dos-as-Graduandos-as-das-IFES-2018.pdf>. Acesso em: 03 set. 2019. 
BRASIL. Ministério da Saúde. Lista Nacional de Medicamentos Essenciais. Brasília: Ministério da Saúde, 2017. Disponível em: <http://apps.who.int/medicinedocs/documents/s23383pt/ s23383pt.pdf>. Acesso em: 02 set. 2019.

- Ministério da Saúde. Secretaria de Ciência, Tecnologia e Insumos Estratégicos. Departamento de Assistência Farmacêutica e Insumos Estratégicos. Assistência farmacêutica na atenção básica: instruções técnicas para sua organização (Série A. Normas e Manuais Técnicos). 2. ed. Brasília: Ministério da Saúde, 2006. Disponível em: <http://bvsms.saude. gov.br/bvs/publicacoes/assistencia_farmaceutica_atencao_basica_instru coes_tecnicas.pdf >. Acesso em: 08 set. 2019.

- Ministério da Saúde. Secretaria de Ciência, Tecnologia e Insumos Estratégicos. Departamento de Assistência Farmacêutica e Insumos Estratégicos. Política Nacional de Medicamentos. Brasília: Ministério da Saúde, maio 2001. Disponível em: <http://bvsms.saude. gov.br/bvs/publicacoes/politica_medicamentos.pdf>. Acesso em: 06 set. 2019.

Ministério da Saúde. Secretaria de Ciência, Tecnologia e Insumos Estratégicos Departamento de Assistência Farmacêutica e Insumos Estratégicos. Uso Racional de Medicamentos. Série A. Normas e Manuais Técnicos. Brasília: Ministério da Saúde, 2012. Disponível em: <http://bvsms.saude.gov.br/bvs/publicacoes/uso_racional_medicamentos_ temas_selecionados.pdf>. Acesso em: 08 set. 2019.

Ministério da Saúde. Secretaria de Ciência, Tecnologia e Insumos Estratégicos. Departamento de Gestão e Incorporação de Tecnologias em Saúde. Entendendo a Incorporação de Tecnologias em Saúde no SUS: como se envolver. Brasília: Ministério da Saúde, 2016. Disponível em: <http://bvsms.saude.gov.br/bvs/publicacoes/entendendo_incorporacao_tecnologias_sus_ env olver.pdf $>$. Acesso em: 09 set. 2019.

- Ministério da Saúde. Secretaria de Ciência, Tecnologia e Insumos Estratégicos. Departamento de Assistência Farmacêutica e Insumos Estratégicos. Uso de Medicamentos e medicalização da vida: recomendaçóes e estratégias. Brasília: Ministério da Saúde, 2019. Disponível em: <https://portalarquivos2.saude.gov.br/images/pdf/2019/fevereiro/15/LivroUSO-DE-MEDICAMENTOS-E-MEDICALIZACAO-DA-VIDA--1-.pdf >. Acesso em: 15 set. 2019.

- Ministério da Saúde. Secretaria de Ciência, Tecnologia, Inovação e Insumos Estratégicos em Saúde. Departamento de Assistência Farmacêutica e Insumos Estratégicos. Relação Nacional de Medicamentos Essenciais: Rename 2020. Brasília: Ministério da Saúde, 2020. 217 p. Disponível em: <http://bvsms.saude.gov.br/bvs/publicacoes/relacao_medicamentos_ rename_2020.pdf>. Acesso em: 06 set. 2020.

CABRITA, J. et al. Estudo do padrão de consumo de medicamentos pelos estudantes da Universidade de Lisboa. Revista Portuguesa de Saúde Pública, v. 19, n. 2, p. 39-47, 2001. 
CAPUCHO, H. C. Monitoramento e Avaliação Farmacoterapêutica: o medicamento fez efeito? Qual? Uso racional de medicamentos: fundamentação em condutas terapêuticas e nos macroprocessos da Assistência Farmacêutica. Brasília, v. 1, n. 20, p. 1-7, 2016.

CAVALCANTI, G. A. O. et al. Efeitos colaterais da ranitidina aplicada em dose terapêutica em cães saudáveis. Ciência Rural, Santa Maria, v. 40, n. 2, p. 356-359, 2010.

DIENER, H. C.; SCHNEIDER, R.; AICHER, B. Per-capita consumption of analgesics: a nine-country survey over 20 years. J. Headache and Pain, v. 9, p. 225-231, 2008.

FARIAS, M. R. et al. Assistência Farmacêutica no Brasil: Política, Gestão e Clínica. Seleção de medicamentos. Florianópolis, v. 3, p. 22-191, 2016.

JACOMINI, L. C. L.; SILVA, N. A. Interaçóes medicamentosas: uma contribuição para o uso racional de imunossupressores sintéticos e biológicos. Rev. Bras. Reumatol, v. 51, n. 2, p. 161-174, 2011.

LEE, S. H. et al. Income Disparities in the Use of Health Screening Services Among University Students in Korea: A Cross-Sectional Study of 2479 Participants in a University. Medicine (Baltimore), v. 95, n. 20, e3681, 2016.

LIMA, T. A. M. et al. Análise de potenciais interaçóes medicamentosas e reaçóes adversas a anti-inflamatórios não esteroides em idosos. Rev. Bras. Geriatr. Gerontol., Rio de Janeiro, v. 19, n. 3, p. 533-544, 2016.

LOPES, E. M.; CARVALHO, R. B. N.; FREITAS, R. M. Análise das possíveis interaçóes entre medicamentos e alimento/nutrientes em pacientes hospitalizados. Einstein, v. 8, n. 3, p. 298-302, 2010.

MANZI, A. L. et al. Guia de medicamentos. Centro Universitário de Araraquara, 2011. Disponível em: https:/www.uniara.com.br/arquivos/file/cursos/graduacao/farmacia/guias-demedicamentos/guia-medicamentos.pdf. Acesso em: 16 set. 2019.

MARQUES, D. C.; ZUCCHI, P. Comissôes farmacoterapêuticas no Brasil: aquém das diretrizes internacionais. Rev Panam Salud Publica, v. 19, n. 1, p. 1-6, 2006.

MLYNARZ-HIDER, K.; CAVALIÉ, P.; MAISON, P. Trends in analgesic consumption in France over the last 10 years and comparison of patterns across Europe. Br J Clin Pharmacol., v. 84, n. 6, p. 1324-1334, 2018.

MONJE, B. et al. Tendências no consumo hospitalar de analgésicos após a implantação de plano de melhoria do controle da dor. Revista Brasileira de Anestesiologia. Campinas, v. 69, n. 3, p. 259-265, 2019.

NASCIMENTO JUNIOR, J. M. et al. Avanços e perspectivas da RENAME após novos marcos legais: o desafio de contribuir para um SUS único e integral. Revista Eletrônica Gestão \&. Saúde, v. 6, n. 4, p. 3354-71, 2015. 
NUNES, A. A. et al. Perfil epidemiológico de usuários de anti-inflamatórios não esteroidais em uma farmácia de dispensação. Revista Interdisciplinar de Ciências Médicas, Teresina-PI, 2016. Disponível em: <https:/gpicursos.com/interagin/gestor/uploads/trabalhos-feirahospitalarpiaui /2ae09a5633a03d2e69ddf3eb973455cb.pdf>. Acesso em: 23 set. 2019.

OLSON, K. R. et al. Manual de toxicologia clinica. New York: The McGraw-Hill, Global Education Holding, 2012.

PINHEIRO, R. M.; WANNMACHER, L. Uso racional de anti-inflamatórios não esteroides. HÓRUS - Sistema Nacional de assistência farmacêutica. Brasília: OPAS, 2010. Disponível em: $<$ https://www.paho.org/bra/index.php?option=com_docman\&view=download\&alias=1317uso-racional-anti-inflamatorios-nao-esteroides-uso-racional-medicamentos-temas-selecionadosn-5-7\&category_slug=assistencia-farmaceutica-958\&Itemid=965>. Acesso em: 16 set. 2019.

PONTES JUNIOR, D. M. et al. A definição de medicamentos prioritários para o monitoramento da qualidade laboratorial no Brasil: articulação entre a vigilância sanitária e a Política Nacional de Medicamentos. Cad. Saúde Pública, v. 24, n. 9, p. 2081-2090, 2008.

PREFEITURA DE SEROPÉDICA. Plano Municipal de Saúde de Seropédica/RJ 2014-2017. p 2-158. Seropédica, 2014. Disponível em: <http://transparencia.seropedica.rj.gov.br/wp-content/ uploads/2017/09/Plano-Municipal-de-Sa\%C3\%BAde-de-Serop\%C3\%A9dica-2014-2017. pdf>. Acesso em: 6 set. 2019.

RATTI, B. A. et al. Soro fisiológico: potencial risco de perda da estabilidade após aberto e armazenado por trinta dias em diferentes meios. In: Encontro Internacional de Produçáo Científica Cesumar., 7. Paraná, p. 1-4, 2011. Disponível em: http://www.cesumar.br/prppge/ pesquisa/epcc2011/anais/bianca_altrao_ratti\%20(2).pdf. Acesso em: 10 set . 2019.

RIBOLDI E.; LIMA, D. A.; DALLEGRAVE, E. Sensibilidade espécie-específica aos antiinflamatórios não esteroidais: humanos $\mathrm{X}$ animais de companhia. Arq. Bras. Med. Vet. Zootec., v. 64, n. 1, p. 39-44, 2012.

SFORSIN, A. C. P. et al. Gestão de compras em farmácia hospitalar. Pharmacia Brasileira, n. 85, p. 3-30, 2012.

SILVA, A. G.; FUZIOKA, P. U.; RIBEIRO NETO, L. M. Complicaçóes clínicas induzidas pelo uso de dipirona (metamizol): estudo sobre os riscos, os benefícios e o seu uso racional. In: Simpósio de Ciências Farmacêuticas., 6. Centro Universitário São Camilo. São Paulo, out. 2015. Disponível em: http://www.saocamilo-sp.br/novo/eventos-noticias/simposio/15/SCF015_15. pdf. Acesso em: 16 set. 2019.

SILVA, H. P.; ELIAS, F. T. S. Incorporação de tecnologias nos sistemas de saúde do Canadá e do Brasil: perspectivas para avanços nos processos de avaliação. Cadernos de Saúde Pública, n. 35, supl. 2, e00071518, 2019. 
SINITOX. Casos Registrados de Intoxicação Humana por Agente Tóxico e Trimestre. Brasil, 2016. Disponível em: <https://sinitox.icict.fiocruz.br/sites/sinitox.icict.fiocruz.br/files//Brasil5_1. pdf $>$. Acesso em: 19 set. 2019.

SOUZA, T. R. et al. Método Dáder de Seguimento Farmacoterapêutico, 3 ed (2007): um estudo piloto. Rev Ciênc Farm Básica Apl. Recife, v. 30, n. 1, p. 105-109, 2009.

SPINKS, A. et al. Scopolamine (hyoscine) for preventing and treating motion sickness. Cochrane Database of Systematic Reviews, 3 ed. Art. n: CD002851, p. 3-24, 2007.

TIERLING, V. L. et al. Nível de conhecimento sobre a composição de analgésicos com ácido acetilsalicílico. Revista de Saúde Pública. São Paulo, v. 38, n. 2, p. 223-227, 2004.

TODESCATO, J. T.; BERTOLOTO, M. I.; MELLO, A. R. N. Classificação terapêutica como facilitadora do uso racional de medicamentos. Revista Cientifica Semana Acadêmica. Fortaleza, 2017. Disponível em: <https://semanaacademica.org.br/artigo/classificacao-terapeutica-comofacilitadora-do-uso-racional-de-medicamentos>. Acesso em: 17 set. 2019

TORRES, R. M. et al. Adesão às Listas de Medicamentos Essenciais por Médicos Brasileiros em Atuação no Sistema Único de Saúde. Revista Brasileira de Educação Médica, v. 38, n. 3, p. 323-330, 2014.

UNIVERSIDADE FEDERAL RURAL DO RIO DE JANEIRO. Deliberação $n^{\circ}$ 51, de 22 de abril de 1981. Regimento da Reitoria e dos Órgãos Subordinados. Seropédica-RJ, 1981.

VALE, N. Desmistificando o uso da dipirona. In: CAVALCANTI, I. I.; CANTINHO, F. A. F.; ASSAD, A. (Orgs.). Medicina Perioperatória. Rio de Janeiro: Sociedade de Anestesiologia do Estado do Rio de Janeiro, 2006. p. 1110-1111.

VASCONCELlOS, M. C.; DUARTE, M. A.; MACHADO, M. G. P. Vômitos: abordagem diagnóstica e terapêutica. Rev Med Minas Gerais, v. 24, supl 10, p. 5-47, 2014.

VIDOTTI, C. C. F. Sistema de Classificação Anatômico Terapêutico Químico (ATC). Centro de Informação sobre Medicamentos. Conselho Federal de Farmácia. INFARM, v. 2, n. 6, p. 1-4, 1993.

VIEIRA, M. C. Doenças típicas de outono e inverno: como manter seu filho longe do prontosocorro. Revista Crescer, São Paulo, 03 abr. 2017. Disponível em: <https://revistacrescer.globo. com/Criancas/Saude/noticia/2017/04/doencas-tipicas-de-outono-e-inverno-como-manter-seufilho-longe-do-pronto-socorro.html>. Acesso em: 03 set. 2019.

VOSGERAU, M. Z. S. et al. Consumo de medicamentos entre adultos na área de abrangência de uma Unidade de Saúde da Família. Ciênc. saúde coletiva, v. 16, supl. 1, p. 1629-1638, 2011. WANNMACHER, L. Medicamentos essenciais: vantagens de trabalhar com este contexto. Uso Racional de Medicamentos: temas selecionados, v. 3, n. 2, p. 1-6, 2006. 
WEBER, D. et al. Seleção de medicamentos: uma visão do processo em quatro municípios do Rio Grande do Sul. Rev. Bras. Farm., v. 9, n. 3, p. 141-8, 2010.

WORLD HEALTH ORGANIZATION. Collaborating Centre for Drug Statistics Methodology. Guidelines for ATC classification and DDD assignment 2020. Oslo, Norway, 2019. 249p. Disponível em: <https://www.whocc.no/filearchive/publications/2020_guidelines_web. pdf>. Acesso em: 06 jul. 2020.

Perspectivas politicas sobre medicamentos de la OMS. Selección de medicamentos esenciales. Ginebra: WHO, 2002a.

WHO Policy Perspectives on Medicines - Promoting rational use of medicines: core components. Geneva: WHO, 2002b.

\section{Nota}

1 J. R. B. dos Santos e P. S. G. de Castro participaram da concepção do trabalho, levantamento de dados, elaboração e revisão do manuscrito. 


\section{Abstract}

Profile of drug selection and consumption in a higher education institution: situational diagnosis for health promotion

Drug selection is an interdisciplinary process based on the local epidemiological, economic and technical profile. The selected drugs must be effective and safe, in accordance with the National Drug Policy. This study aimed to identify the profile of selection and consumption of drugs dispensed in a higher education institution. From descriptive research with quantitative and qualitative characteristics, drug dispensing maps for the Nursing Division of the Health Division of 2018 and 2019 were used. In 2018, 21\% of standardized medicines were not consumed. For this reason, in 2019 there was a new selection and $17.2 \%$ of medicines were excluded. According to the medication consumption profile, the consumption of analgesics was higher than the consumption of drugs with anti-inflammatory purposes, according to the Anatomical Therapeutic Chemical (ATC) classification. It is concluded that the profile addressed in this study allows a situational diagnosis of selection and consumption, with the need to implement pharmacotherapeutic follow-up in order to minimize the possible adverse reactions produced by drugs, as a health promotion strategy.

> Keywords: drug evaluation; drugs essential; drug utilization; health promotion. 